\title{
A FITNESS BIKE MAINTENANCE TRAINING SYSTEM WITH THREE-DIMENSIONAL COMPUTER GRAPHICS
}

\author{
Takayuki Fujiwara ${ }^{1}$, Shintaro Tsuchiya ${ }^{1}$, Norihisa Komoda ${ }^{2}$ and Toru Fujiwara ${ }^{2}$ \\ ${ }^{1}$ Research \& Development Division, Hitachi Systems, Ltd. 1-2-1, Osaki, Shinagawa, Tokyo, 141-8672, Japan \\ ${ }^{2}$ Graduate School of Information Science and Technology, Osaka University, \\ 1-5, Yamadaoka, Suita, Osaka, 565-0871, Japan
}

\begin{abstract}
This article describes a fitness bike maintenance training system using 3D computer graphics. The proposed system provides customer engineers with a 360-degree view and enables interactive manipulation. The system has three training modes: learning mode, examination mode, and positional relation check mode. Flashing CG objects and interactive manipulation are used so that engineers can readily understand the maintenance work. Test usage by customer engineers, managers, and training instructors has resulted in several positive comments including praise for the clearly indicated instructions and good instructions for handling the bike in 360-degrees.
\end{abstract}

\section{KEYWORDS}

Maintenance, Training, CAE, 3D, Fitness bike, E-learning

\section{INTRODUCTION}

Fitness bikes are widely used for exercise. These are installed in sports and fitness clubs nationwide. They require not only periodic maintenance to prevent faults but also fixing failure. Customer engineers working at numerous maintenance bases have to learn the structure, disassembly procedures, and troubleshooting skills for these bikes. Traditionally, the training is generally done by reading manuals or watching video tutorials. Training with actual machines is not always used because of two reasons. The first reason is that actual machines are not provided at all maintenance bases. Engineers at some maintenance bases cannot receive maintenance training with actual machines. Another reason is that engineers do not have enough time for training because they are constantly being sent out to fulfill maintenance requests. Additionally, the instruction pictures or figures in the training materials are always shown from single angle, such that engineers sometimes confuse the positions and viewpoints. Therefore, it is difficult to develop specific images of the corresponding machine and parts. Furthermore, both training methods do not include user manipulation for training but just reading or watching. They also do not include a method for confirming understanding of procedures and precautions.

To solve these problems, Computer Aided Engineering (CAE) is effective. Three-dimensional computer graphics (3DCG) of the fitness bike are effective in grasping an image of the corresponding machine. 3DCG has been studied for product design (Kuang, 2014), and has been adapted for assembly simulation (Flavien, 2012), and assembly training (Zhilong, 2013; Terasa, 2010). 3DCG and Augmented Reality based maintenance training system has also been studied (Francesca, 2011; Sabine, 2011; Michele, 2014). However, engineers need actual machines to use these AR-based maintenance training systems. 3DCG and virtual reality-based maintenance training systems have also been studied (Sportillo, 2015). However, since engineers need high performance PC, VR headsets, tracking devices, and large spaces, it is difficult for all customer engineers to prepare devices and spaces because they are usually busy with maintenance service calls.

In this paper, we propose a tablet or laptop PC based system using 3DCG for fitness bike maintenance training. Then, the effects of the combination of 3DCG and instructional text, and the proposed user interface are evaluated through testing. 


\section{MAINTENANCE TRAINING}

\subsection{Fitness bike}

Fitness bikes are installed at sports clubs nationwide for health enhancement. When a bike has a problem, clubs ask maintenance personnel to repair it.

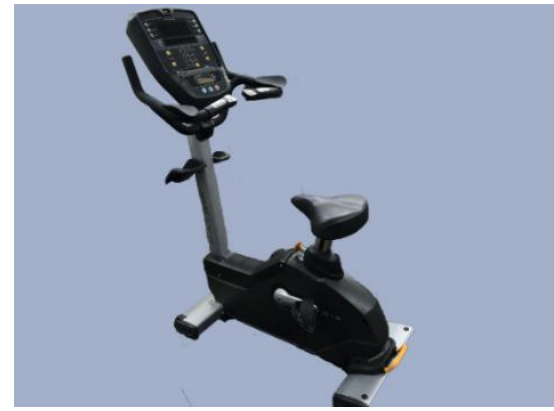

Figure 1. Overview photo of the target fitness bike

A fitness bike shown in Figure 1 is our target fitness bike. It consists of more than twenty parts including electronic parts. Customer engineers are stationed at several maintenance bases. They must learn how to disassemble the bike, how to make a failure diagnosis, how to fix the bike, and how to re-assemble the bike before engaging in any bike maintenance tasks.

\subsection{Traditional training Methods for Fitness Bike}

Fitness bikes are only prepared on a limited basis. Therefore, traditionally, printed manuals and video are used for maintenance training of the fitness bike. Manuals show how to disassemble the bike and precautions so that parts are not damaged. However, it is not always easy for engineers to understand it because some instructions do not include pictures or figures. The manual of the fitness bike has over 50 pages with 70 pictures. It is not sufficient because it does not include all troubleshooting instructions.

Video tutorials teach disassembly procedures with the instructor's movements. These movements are effective in getting engineers to understand the disassembly procedures. However, video tutorials also present difficulties for engineers in understanding positional relations. For example, when a video tutorial describes how to replace a circuit board in the fitness bike, it zooms in on the board or instructor's hands. Engineers sometimes become confused as to whether the board is set on left or right side. Furthermore, just watching videos is a monotonous and time-consuming work as the videos are a few hours long. It is difficult to learn all troubleshooting instructions with such a monotonous learning method.

\subsection{Requirements of Maintenance Training System for Fitness Bike}

Engineers have already used tablets or laptop PCs for maintenance work. Therefore, the training system should also run on these devices. By usage of hardware used in daily maintenance work, the engineers can learn during their fragmented free time such as while travelling or waiting.

From the viewpoint of software, engineers should be able to check target machines from all angles. If engineers read an instruction "Remove the left cover", they might be confused about to which "left" it actually refers. Engineers should also be able to check maintenance procedures through their own actual handling of the bike. With only boring reading manuals or watching videos, it is difficult to understand the procedures. 


\section{MAINTENANCE TRAINING CONTENT FOR FITNESS BIKE}

\subsection{User Interface of the System}

The main display of the training content is shown in Figure 2. The display is segmented into three area in order to easily understand the training process.

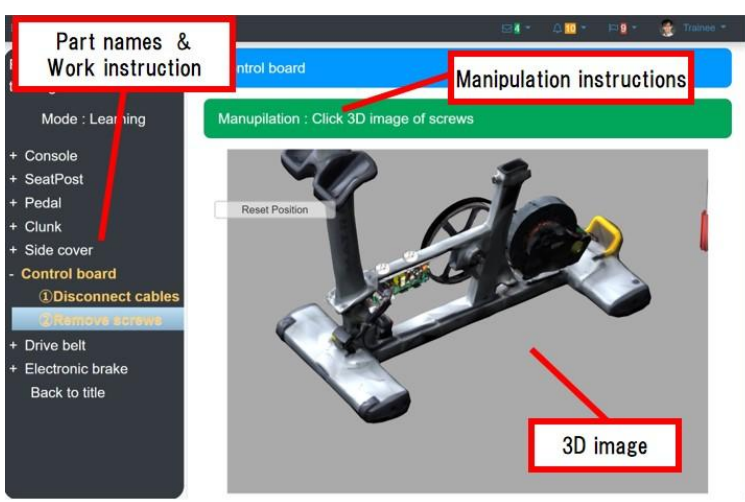

(a) An example of control board disassembly

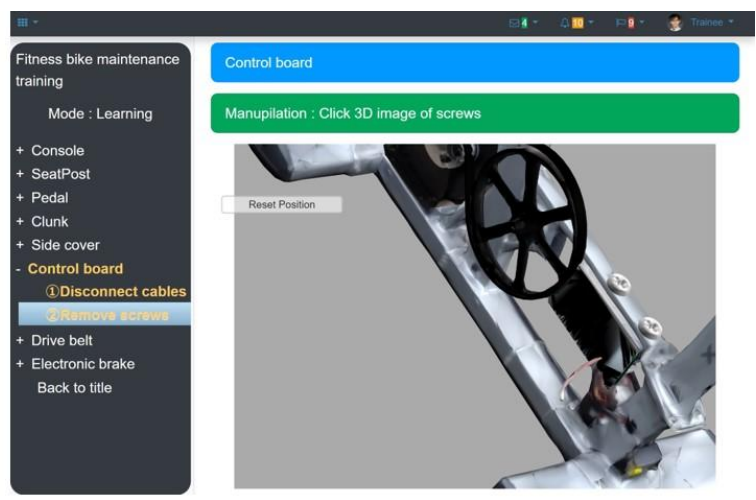

(b) An example of another angle

Figure 2. Main screen shots of the content

(1) Part names and work instructions area

The left area of the display is for part names and the work instruction area shown in Figure 2(a). All part names of the machine and all work instructions are displayed here. The fitness bike parts are a pedal, saddle, cover, console panel, belt, etc. The tree-view index has been designed according to the requests of engineers. For example, when engineers click the part name of "Control board", work instructions such as "(1) Disconnect cables" and "(2) Remove screw” are displayed.

(2) Manipulation instructions area

The upper right area of the display is a manipulation instructions area as shown in Figure 2(a). The messages displayed in this area are manipulation instructions for each step. Engineers can readily understand maintenance procedures through virtual manipulation just by following the instructions. The area is also designed based on engineers' requests. Clear manipulation instructions are required because engineers are not familiar with the maintenance training system.

(3) 3D image area

The large area at the lower right is the content area. A 3D image of the fitness bike is displayed shown in Figure 2(a). In the area, parts that engineers should select flash in yellow. Engineers can see the fitness bike from a 360-degree angle by using the mouse or screen-touch. For example, when engineers rotate and zoom the fitness bike, the resulting image is displayed in this area as shown in Figure 2(b).

\subsection{Training Modes}

The training modes are classified as (1) learning mode, (2) examination mode, and (3) positional relation check mode from the results of an analysis of training manuals and videos.

\subsubsection{Learning Mode}

Engineers should learn step by step how to disassemble the machine, the positions of parts, and precautions. In learning mode, engineers can check every disassembly step with clear instructions. The main screenshot and sequence are shown in Figure 3.

As shown in Figure 3(a), a text instruction message flashes in the first step. In the example of replacing a drive belt in Figure 3(b), the first message is "(1) Loosen the screw of belt tension". After engineers click the text, the 3D image of the three screws flashes. Clicking the screws causes them to rotate and move. When the second message "(2) Loosen the belt pulley" in Figure 3(b) is clicked, a wrench approaches the three screws. When the third message "(3) Remove the drive belt" in Figure 3(b) is clicked, a warning dialog box is 
displayed as shown in Figure 3(b). Engineers must learn the precaution messages for some parts. For example, when replacing a drive belt of the fitness bike, engineers might have their fingers pinched between the wrench and belt if distracted. In learning mode, the procedures cannot advance without manipulation on the part of the engineer. Through their step-by-step clicking and confirmation, engineers can easily and deeply understand the maintenance procedures. They can learn each operation and the corresponding important points and precautions.

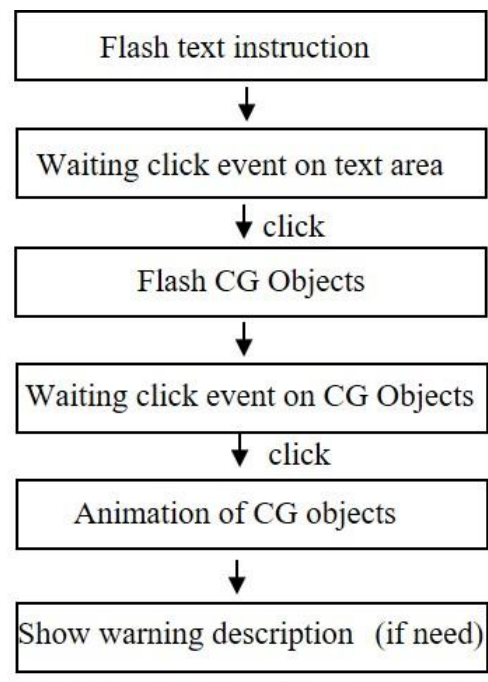

(a) Main sequence of learning mode

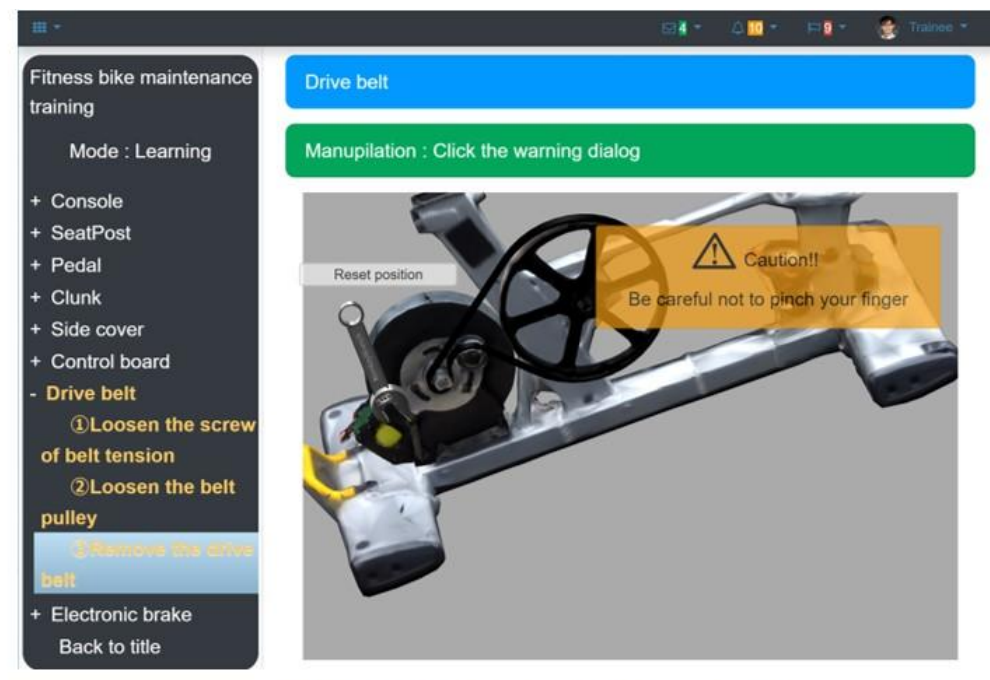

(b) An example of warning description

Figure 3. Main sequence and screenshot of learning mode

\subsubsection{Examination Mode}

Engineers should confirm their comprehension of the machine itself and corresponding maintenance procedures. In examination mode, engineers take comprehension tests about the corresponding parts, positions, and directions. The example screenshot and sequence are shown in Figure 4.

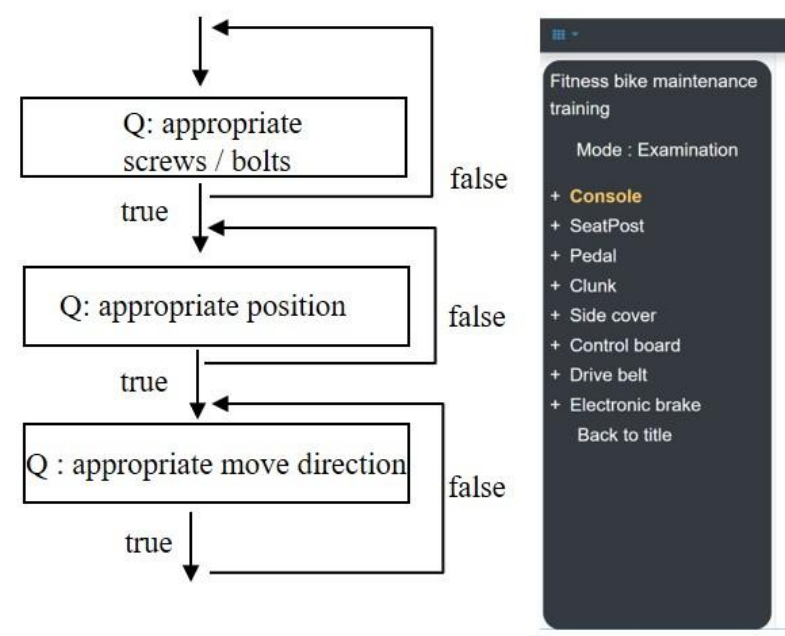

(a) Main sequence of examination mode

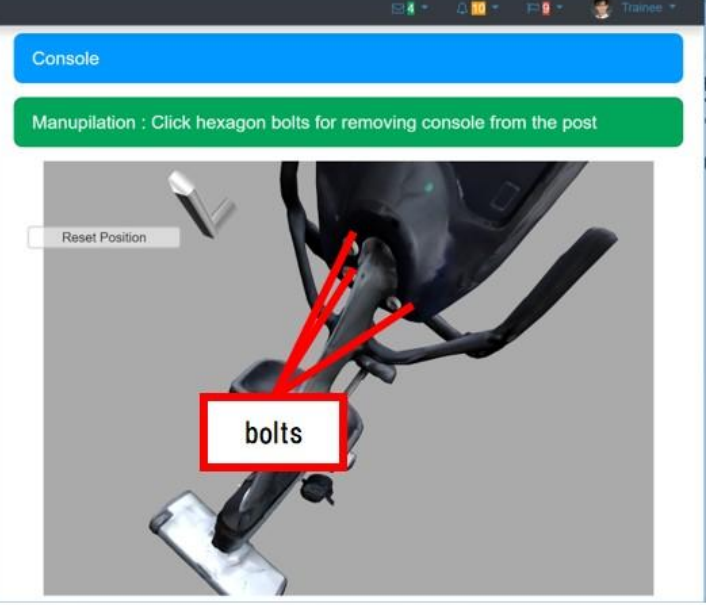

(b) An example of appropriate bolts

Figure 4. Main sequence and screenshot of examination mode

As shown in Figure 4(a), engineers select the appropriate screws or bolts, and they click or tap the appropriate 3D images of bolts in Figure 4(b). If the answer is wrong, they must try again. After engineers select the appropriate bolts, the 3D image of the hexagonal wrench is rotated, and the bolt is removed. 
When removing a console panel from a fitness bike's post, engineers must prop the console panel against a grip of the bike. They must not remove the console panel completely because it has several electronic cables. Engineers must be careful to protect the grip of the bike when propping up the console panel. Propping up the panel might scratch the grip. As shown by the "appropriate position" in Figure 4(a), engineers select the appropriate propped position. In this example, it is correct when they click or tap the area marked by the red square as shown in Figure 5(a). After engineers click or tap the appropriate position, the protective covering sheet moves close to the grip.

Engineers must be careful to remove parts gradually. In the example of the console panel removal, it must be removed in two steps. As shown by the "appropriate move direction" in Figure 4(a), engineers select the appropriate direction in the first step as shown in Figure 5(b). All directional objects are 3D image. If engineers correctly click or tap in the up direction in the 3D image, the 3D image of the console panel is moved up to lean against the grip of the bike.

This step-by-step checking of appropriate parts, positions, and movement directions checks whether the costumer engineers understand how to disassemble parts.

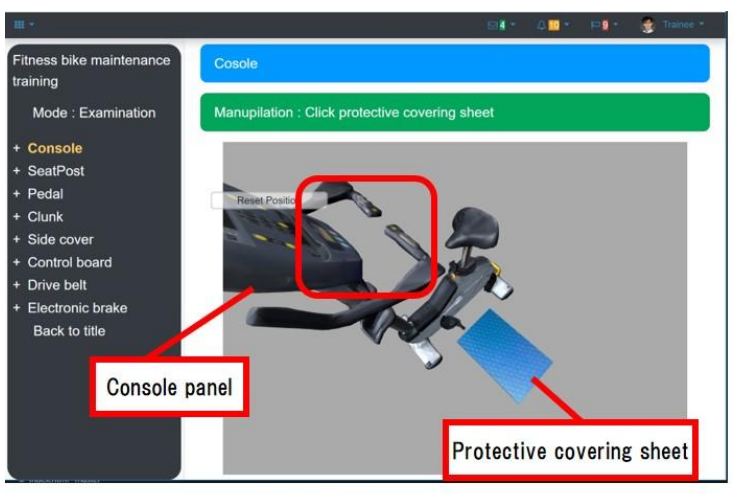

(a) An example of selecting appropriate position

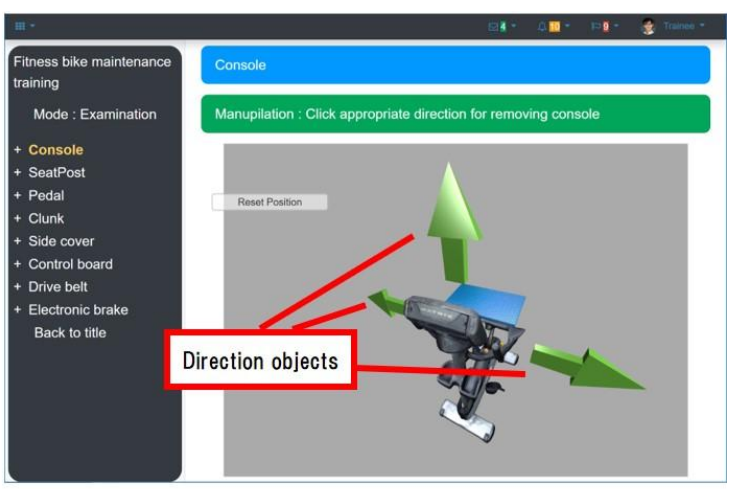

(b) An example of selecting appropriate direction

Figure 5. An example of selecting direction test

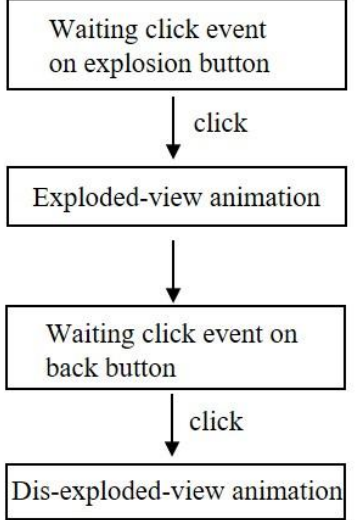

(a) Main sequence of positional relation check mode

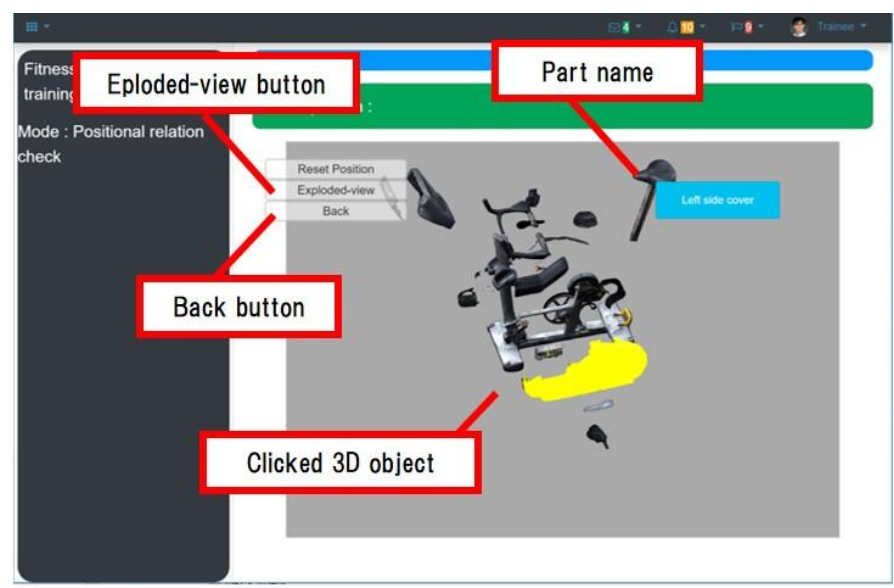

(b) An example view of positional relation check mode

Figure 6. Main sequence of check mode

\subsubsection{Positional Relation Check Mode}

Engineers should learn positional relations and part names of the machines. In the positional relation check mode, engineers check an exploded-view animation for all parts that provides engineers with the positional relation of each part. The main screenshot and sequence are shown in Figure 6.

As shown in Figure 6(a), the engineers click or tap the exploded-view button. The result is shown in Figure 6(b). All parts are spread out radially, and engineers can check all parts from a 360-degree angle. The color of a clicked 3D object is changed to yellow and the name is shown in a blue square. For example, the 
clicked object in Figure 6(b) is the left cover. When engineers click the back button, all parts return to their initial positions. Use of this clearly indicated exploded-view animation allows engineers to intuitively understand the positional relations of parts.

\section{SYSTEM STRUCTURE}

The software system structure of the training system is shown in Figure 7. Training content is consisted of program, data definition files, and media data. Files in data definition files define content data as 3D models, sequence, and instruction text. Media data includes 3D model. Common parts in the data are tools or parts that used at maintenance work commonly. A driver, wrench, and spanner are example of the tools. A screw, bolt, and nut are example of the parts. Program parses data definition files and displays the content. A layout file in the program defines content layout as shown in Figure 2.

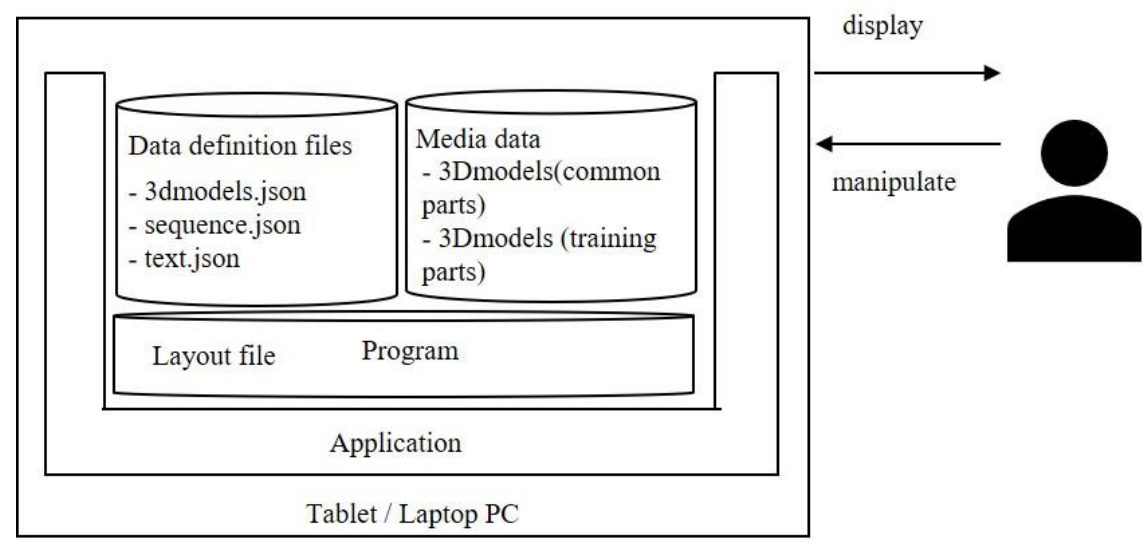

Figure 7. System structure

A simple example of the relation between data definition and display are shown in Figures 9, 10, and 11. Areas (1) to (11) in Figure 8 consist of (1) to (11) in Figures 10 and 11. Figure 8 shows maintenance training for the drive belt. The training has three sequences. Figure 8(a) shows the third sequence as "(3) Remove the drive belt" shown in Figure 8. In the third sequence, a warning message "Be careful not to pinch your finger" is overwritten on the 3D image in the content area. When engineers click the caution box, the dialog disappears. Then, the next and forth sequence is displayed on devices as shown in Figure 8(b).

These sequences are managed by the data definition files. All of the data definition files as shown in Figures 10(a), (b), Figure 10(a), and (b) consists of JSON (JavaScript Object Notation). JSON is a format to manage data using keys and values (ECMA, 2017). For example, "workItemAreaId" in Figure 9(a) is a key, with "wi7" being a value. (1), (2), and (3) in Figure 9(a) define the text of the content. (4) and (5) in Figure 9 (b) define the sequence number and conditions of proceeding on to the next sequence. Each segment of (4) and (5) defines each sequence.

"clickedText_id" of (4) in Figure 9(b) is a condition for proceeding to the next sequence. "clicked3dObject_id" of (4) in Figure 9(b) is not a condition because the value is blank. A layout file as shown in Figure 9(c) has attribute IDs. Each ID is related to an ID in "sequence.json" as shown in Figure 9(b). The tags as "workItemArea", "manipulationArea", and "3DimageArea" in Figure 9(c) are all rendered by the program in Figure 7. "clickedText_id" of (4) in Figure 9(b) is "mA7-3w". "mA7-3w" is the same as (2) in Figure 9(a). Therefore, the content is changed from (a) to (b) in Figure 8 when engineers click the warning text dialog of (2) in Figure 8(a). (5) in Figure 9(b) shows that "clickedObject_id" is "tp-7". "tp-7"is an identifier of the 3D model. An example definition of the 3D model by JSON is shown in Figure 10. (6) in Figure 10(a) defines a 3D wrench object. It is an example of common parts. Common parts include tools such as screwdrivers and wrenches." objFilePath" in Figure 10 is a path to the file in OBJ format, a commonly used 3D data format. It includes vertex, and surface information of a 3D object. "mtlFilePath" indicates is a path to the file in MTL format, a support format of OBJ. It manages the texture path and color information of the OBJ file. Figure 10(a) manages position, rotation and scale of the 3D wrench object. (7) in Figure 10(b) defines the body of the 3D object of the fitness bike. (8) in Figure 10(b) defines the 3D drive belt object of the fitness bike. These are training parts. 


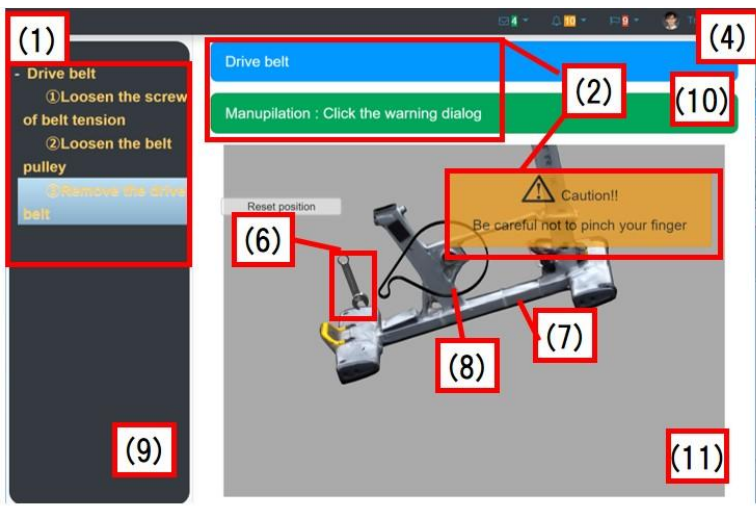

(a) An example of sequence 3

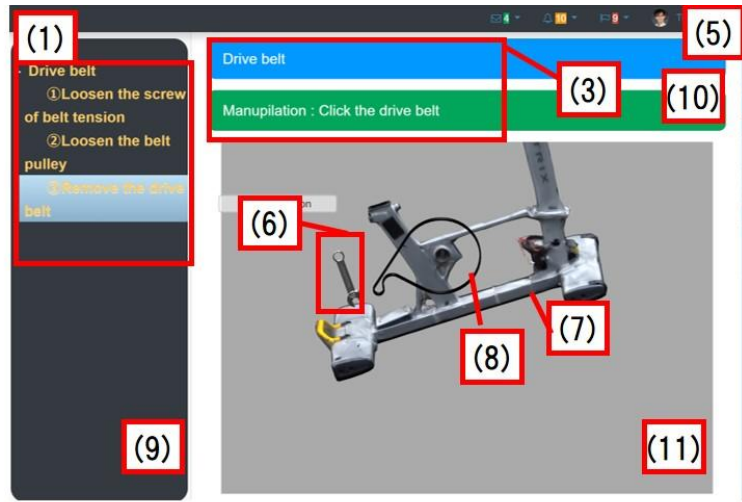

(b) An example of sequence 4

Figure 8. Example of display of sequence 3 and 4

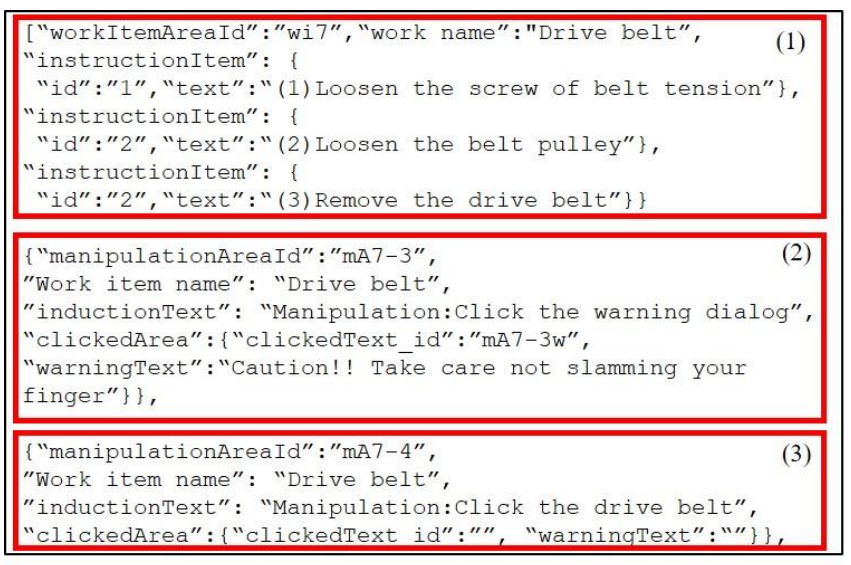

(a) An example of work item and instruction in text.json

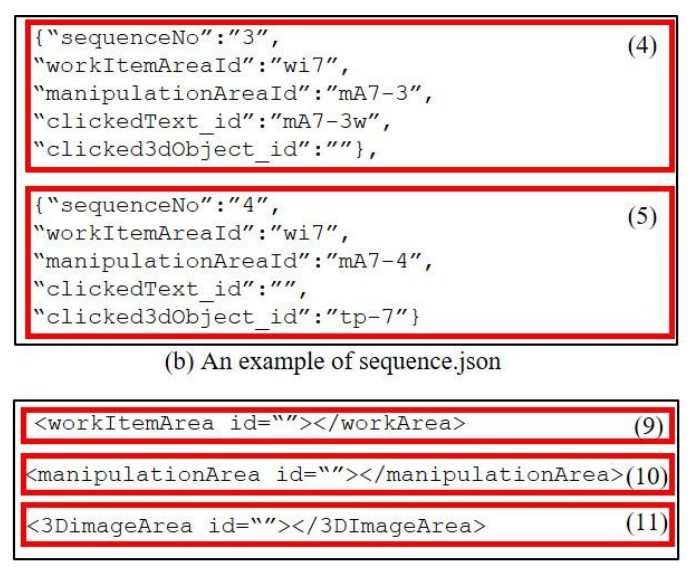

(c) An example of layout file

Figure 9. Example of data definition files

\section{EVALUATION}

Several customer engineers, mangers and training instructors carried out trial operation of our prototype system. We provided the content with through use of their laptop PC and tablet.

With our proposed system, examinees can learn each training content in less than 10 minutes. It is much more effective than the traditional training method. They can easily check any parts or instructions they want to know. Generally, they said that flashing objects and interactive manipulation enabled them to easily understand how to disassemble the fitness bike. Major comments on each mode were as follows:

(1) Learning mode

In learning mode, engineers can learn step-by-step assembly work by text instruction and flashing parts. Engineers said these instructions are highly effective in understanding what to do next. Instructors said that warning messages promote an understanding of maintenance training.

(2) Examination mode

Engineers said that examination mode is highly effective in making them remember the appropriate tools, part positions, and warning messages. Managers said that they can utilize the results of examinations for skills

management of the engineers.

(3) Positional Relation Check mode

Engineers' instructors said that positional relation check mode is a simple and good feature to check positions and parts names. 
Some of them said difficult manipulation demotivated them to use such a CAE-based system, but our proposed system requires only simple manipulation on a laptop or tablet, and therefore it can be used easily by engineers. It was an important fact to adapt to our proposal for maintenance training.

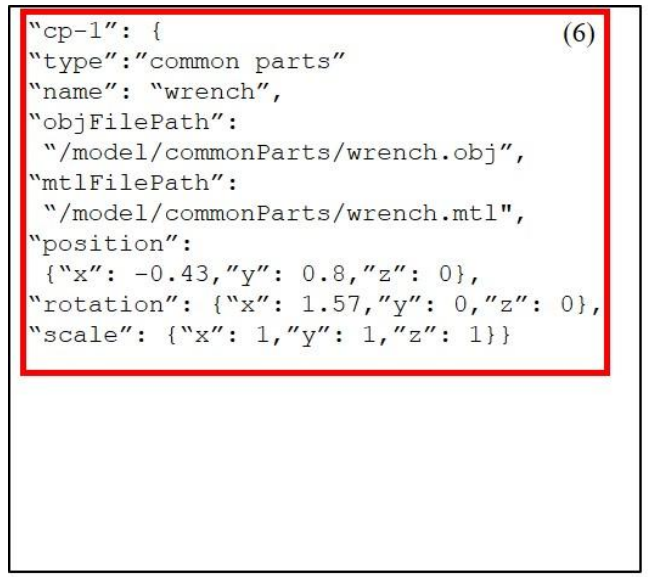

(a) An example of common part in 3dmodels.json

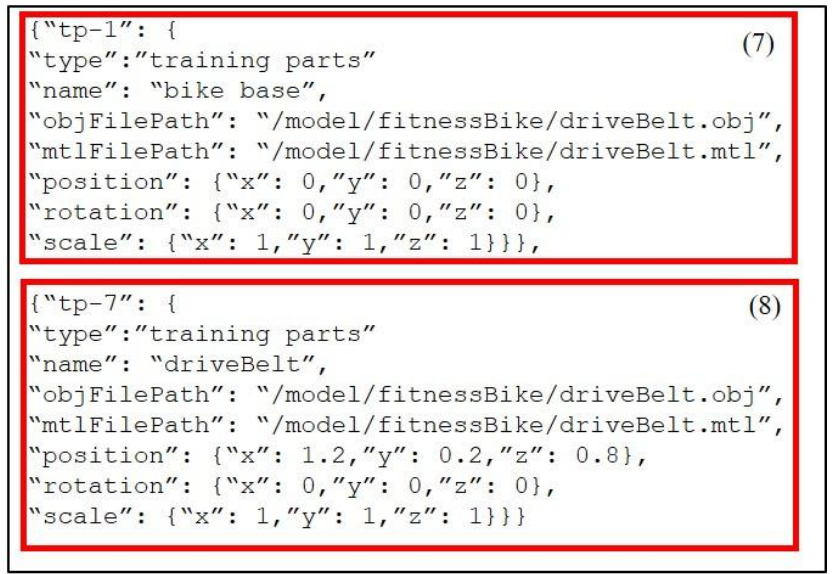

(b) An example of training parts in 3dmodels.json

Figure 10. Example of 3dmodels.json

\section{CONCLUSION}

We developed a maintenance training system using a 3D model for a fitness bike. The system has the three training modes of learning, examination, and positional relation check. Each mode contains intuitive and interactive manipulation and shows step-by-step instructions. The system can easily exchange sequences, text messages and 3D objects with data management in the JSON format. Simple evaluation tests clearly indicated that the proposed maintenance training is effective and useful for training in fitness bike maintenance.

\section{REFERENCES}

Boussuge F. et al, 2012. An analysis of DMU transformation requirements for structural assembly simulations, Proceeding of International Conference of Engineering Computational Technology (ECT), Dubrovnik, Croatia, pp. 1-22.

Chang K, 2014. Product Design Modeling using CAD/CAE. Academic Press, Cambridge, USA.

European Computer Manufacturers Association (ECMA), 2017. The JSON Data Interchange Syntax, Standard ECMA -404, Geneva, Switzerland, pp 1-7.

Fiorentino M, et al, 2014. Augmented reality on large screen for interactive maintenance instructions, Computers in Industry, Vol. 65, No. 2, pp 270-278.

Francesca D, et al, 2011. Augmented Reality for Aircraft Maintenance Training and Operations Support, IEEE Computer Graphics and Applications, Vol. 31, No. 1, pp 96-101.

Gutierrez T. et al, 2010. IMA-VR: A multimodal virtual training systems for skills transfer in Industrial Maintenance and Assembly tasks, Proceeding of International Symposium in Robot and Human Interactive Communication, Viareggio, Italy, pp. 428-433.

Liu Z. et al, 2013. Design and Implementation of Virtual Disassembly and Assembly Training System of Plunger Pump, Proceeding of IEEE International Conference of Virtual Reality and Visualizations, Shaanxi, China, pp. 289-292.

Sportillo D. et al. 2015. Training in VR: A Preliminary Study on Learning Assembly/Disassembly Sequences, Proceeding of International Conference of Augmented and Virtual Reality (AVR), Lecce, Italy, pp 332-343.

Webel S, et al, 2011. Design Criteria for AR-Based Training of Maintenance and Assembly Tasks, Proceeding of International Conference on Virtual and Mixed Reality, Orlando, USA, pp 123-132. 